\title{
miR-335 promotes ferroptosis by targeting ferritin heavy chain 1 in in vivo and in vitro models of Parkinson's disease
}

\author{
XINRONG LI ${ }^{1,2^{*}}$, WENWEN SI $^{1 *}$, ZHAN LI $^{3}$, YE TIAN $^{4}$, XUELEI LIU ${ }^{2}$, SHANYU YE $^{5}$, ZIFENG HUANG $^{1,2}$, \\ YICHUN JI ${ }^{4}$, CAIPING ZHAO ${ }^{2}$, XIAOQIAN HAO ${ }^{1,2}$, DONGFENG CHEN ${ }^{5}$ and MEILING ZHU ${ }^{1}$ \\ ${ }^{1}$ Traditional Chinese Medicine Innovation Research Center, Shenzhen Hospital of Integrated Traditional Chinese and \\ Western Medicine, Shenzhen, Guangdong 518104; ${ }^{2}$ Graduate School, Guangzhou University of Chinese Medicine, \\ Guangzhou, Guangdong 510405; ${ }^{3}$ Guangdong Key Laboratory of Orthopedic Technology and Implant Materials, \\ Key Laboratory of Trauma and Tissue Repair of Tropical Area of PLA, Hospital of Orthopedics, General Hospital of \\ Southern Theater Command of PLA, Guangzhou University of Chinese Medicine, Guangzhou, Guangdong 510010; \\ ${ }^{4}$ Baoan Hospital of Traditional Chinese Medicine Affiliated to Guangzhou University of Traditional Chinese Medicine, \\ Shenzhen, Guangdong 518101; ${ }^{5}$ Department of Anatomy, The Research Center of Basic Integrative Medicine, \\ Guangzhou University of Chinese Medicine, Guangzhou, Guangdong 510006, P.R. China
}

Received July 30, 2020; Accepted February 2, 2021

DOI: 10.3892/ijmm.2021.4894

\begin{abstract}
Parkinson's disease (PD) is a neurodegenerative disease characterized by the selective loss of dopaminergic neurons in the substantia nigra $(\mathrm{SN})$. In a previous study, the authors demonstrated that ferritin heavy chain 1 (FTH1) inhibited ferroptosis in a model of 6-hydroxydopamine (6-OHDA)-induced PD. However, whether and how microRNAs (miRNAs/miRs) modulate FTH1 in PD ferroptosis is not yet well understood. In the present study, in vivo and in vitro models of PD induced by 6-OHDA were established. The results in vivo and in vitro revealed that the levels of the ferroptosis marker protein, glutathione peroxidase 4 (GPX4), and the PD marker protein, tyrosine hydroxylase $(\mathrm{TH})$, were decreased in the model group, associated with a decreased FTH1 expression and the upregulation of miR-335. In both the in vivo and in vitro models, miR-335 mimic led to a lower FTH1 expression, exacerbated ferroptosis and an enhanced
\end{abstract}

Correspondence to: Professor Dongfeng Chen, Department of Anatomy, The Research Center of Basic Integrative Medicine, Guangzhou University of Chinese Medicine, 232 Waihuan East Road, Guangzhou, Guangdong 510006, P.R. China

E-mail: cdf27212@21cn.com

Professor Meiling Zhu, Traditional Chinese Medicine Innovation Research Center, Shenzhen Hospital of Integrated Traditional Chinese and Western Medicine, 3 Shajing Street, Baoan, Shenzhen, Guangdong 518104, P.R. China

E-mail: meilingzhu2020@126.com

*Contributed equally

Key words: miR-335, ferroptosis, ferritin heavy chain 1, Parkinson's disease, rat model
PD pathology. The luciferase 3'-untranslated region reporter results identified FTH1 as the direct target of miR-335. The silencing of FTH1 in 6-OHDA-stimulated cells enhanced the effects of miR-335 on ferroptosis and promoted PD pathology. Mechanistically, miR-335 enhanced ferroptosis through the degradation of FTH1 to increase iron release, lipid peroxidation and reactive oxygen species (ROS) accumulation, and to decrease mitochondrial membrane potential (MMP). On the whole, the findings of the present study reveal that miR-335 promotes ferroptosis by targeting FTH1 in in vitro and in vivo models of PD, providing a potential therapeutic target for the treatment of PD.

\section{Introduction}

Ferroptosis is the process of iron $\left(\mathrm{Fe}^{2+}\right)$-dependent programmed cell death, characterized by $\mathrm{Fe}^{2+}$-dependent lipid peroxidation, restricted metabolism, the decline of mitochondrial membrane potential (MMP) and the accumulation of intracellular reactive oxygen species (ROS) (1-4). A reduced glutathione peroxidase 4 (GPX4) activity (5) is a potent activator of ferroptosis. GPX4 is a crucial regulator of ferroptosis (6), and the inhibition of the function of GPX4 leads to the $\mathrm{Fe}^{2+}$-dependent formation of toxic lipid ROS, resulting in the induction of ferroptosis (7). $\mathrm{Fe}^{2+}$ emits electrons that generate excessive ROS, and $\mathrm{Fe}^{2+}$-dependent lipid oxygenase can promote the oxidation of polyunsaturated fatty acids (PUFAs). Ferritin heavy chain 1 (FTH1) can reverse ferroptosis by regulating iron storage and plays a vital role in proper $\mathrm{Fe}^{2+}$ management required for cell growth and function (8).

FTH1 is a $21-\mathrm{kDa}$ subunit of the ferritin complex, has complementary $\mathrm{Fe}^{2+}$ modulating functions, and forms triple and quadruple pores. FTH1 is primarily regulated at a post-transcriptional level in response to intracellular $\mathrm{Fe}^{2+}$ concentrations. It can trap $\mathrm{Fe}^{2+}$ ions in the cell, and convert them to $\mathrm{Fe}^{3+}$, reducing ROS production (9-11). Thus, FTH1 
is essential for maintaining $\mathrm{Fe}^{2+}$ homeostasis and preventing $\mathrm{Fe}^{2+}$ overload (12). $\mathrm{Fe}^{2+}$ release and ferritin degradation are associated with cell dysfunction through the process of ferritinophagy, causing ROS formation and lipid peroxidation by releasing iron at sufficient levels, triggering ferroptosis (13). In previous studies, the authors demonstrated that was involved in ferroptosis in in vivo and in vitro models of Parkinson's disease (PD) $(14,15)$. Therefore, it is of considerable interest to explore the upstream molecules targeting FTH1.

PD is a chronic neurodegenerative disease. It is associated with a reduction in the neurotransmitter dopamine (DA) in the striatum (in particular, the putamen nucleus) characterized by the loss of tyrosine hydroxylase (TH)-positive nigrostriatal dopaminergic neurons in the substantia nigra (SN). Moreover, the excessive deposition of labile iron in the substantia nigra compact (SNc) has also become a PD pathological mark (16). Iron accumulated in the dopaminergic area of patients with PD is $70 \%$ higher compared with that of healthy individuals (17). The pathogenic changes observed in $\mathrm{PD}$, including nigral iron elevation, mitochondriopathy, glutathione depletion, lipid peroxidation and increased ROS generation are closely associated with ferroptosis (13). However, the underlying mechanisms of ferroptosis in PD remain to be determined.

Ferroptosis, an iron-dependent cell death pathway, has recently been identified to play a role in the early stages of $\mathrm{PD}(15,18) . \mathrm{Fe}^{2+}$ is a potent reducing agent that can generate hydroxyl radicals and cause dopamine oxidation, which can act to further increase the oxidative environment of the SN and contribute to dopaminergic neuron loss (19). Genetic disorders that cause $\mathrm{Fe}^{2+}$ homeostasis in the brain often lead to the development of PD, suggesting that elevated $\mathrm{Fe}^{2+}$ levels contribute to the pathology of the disease $(20,21)$. Moreover, mutations in several proteins related to $\mathrm{Fe}^{2+}$ intake and export are also associated with PD. For example, a mutation in the transferrin protein, a crucial protein for neuronal $\mathrm{Fe}^{2+}$ absorption, is associated with increased PD susceptibility $(22,23)$. This finding suggests that dysregulated $\mathrm{Fe}^{2+}$ homeostasis in $\mathrm{PD}$ contributes to the pathogenesis of the disease. As such, investigating the association between $\mathrm{Fe}^{2+}$ management and the occurrence of ferroptosis may lead to a better understanding of the progression of PD.

MicroRNAs (miRNAs/miRs) are endogenously expressed 22-nucleotide non-coding RNAs. They regulate gene expression following transcription through base pairing with complementary sequences within the 3'-UTR of mRNAs (24-27). Previous studies have indicated that miRNAs play a vital role in the pathogenesis of PD, including dopaminergic neuron degeneration and $\mathrm{Fe}^{2+}$ regulation (28-30). However, few studies have investigated the mechanisms through which miRNAs regulate ferroptosis in $\mathrm{PD}$ by targeting proteins related to $\mathrm{Fe}^{2+}$ management.

In the present study, in vivo and in vitro models of 6-hydroxydopamine (6-OHDA)-induced PD were used to investigate the role of miRNAs in ferroptosis, and to examine the association between FTH1 and the occurrence of ferroptosis in PD. The findings presented herein identify FTH1 as a potential biomarker in PD and further suggest the potential of FTH1 to act as a therapeutic target for the treatment of this chronic neurodegenerative disease.

\section{Materials and methods}

Animals and materials. A total of 48 male Sprague-Dawley rats (weighing, 180-220 g; 6-8 weeks of age) were purchased from the Animal Center of Guangzhou University of Chinese Medicine (Guangzhou, China). All animal procedures were approved by the Ethics Committee of Guangzhou University of Chinese Medicine and followed the regulations of the National Institute of Health Guide for the Care and Use of Laboratory Animals. All animals were acclimated for 7 days prior to surgery and were housed in a standard animal room (humidity, $50-70 \%$; temperature, $21-25 \Delta^{\circ} \mathrm{C}$ ) with a 12 -h light-dark cycle and ad libitum access to food and water.

In vivo model of $P D$. PD was established in the rats as previously described (15). Briefly, the rats were anesthetized $(100 \mathrm{mg} / \mathrm{kg}$ ketamine and $10 \mathrm{mg} / \mathrm{kg}$ xylazine, intraperitoneal injection) and placed in a stereotaxic apparatus with the skull flat. An intracerebral injection of 6-OHDA (cat. no. H116; Sigma-Aldrich; Merck KGaA; $32 \mathrm{mg}$ of 6-OHDA in a total of $4 \mathrm{ml}$ in $0.02 \%$ ascorbic acid saline solution (concentrations of $8 \mathrm{mg} / \mathrm{ml} \mathrm{6-OHDA)} \mathrm{was} \mathrm{performed} \mathrm{at} 2$ sites in the right $\mathrm{SN}$ pars compacta $(\mathrm{SNpc})$ and ventral tegmental area (VTA): Anteroposterior $(\mathrm{A} / \mathrm{P})=-4.9 \mathrm{~mm}$; mediolateral $(\mathrm{M} / \mathrm{L})=-1.9 \mathrm{~mm}$; dorsoventral $(\mathrm{D} / \mathrm{V})=-7.5 \mathrm{~mm}$; and anteroposterior $(\mathrm{A} / \mathrm{P})=-4.9 \mathrm{~mm}$; mediolateral $(\mathrm{M} / \mathrm{L})=-1.1 \mathrm{~mm}$; dorsoventral $(\mathrm{D} / \mathrm{V})=8.0 \mathrm{~mm}$. During the surgery, body temperature was maintained at $\sim 36.5^{\circ} \mathrm{C}$ using a heating pad. All rats received meticulous post-operative care.

Intracerebral injection of mimic and inhibitor. A total of 24 rats were randomly assigned to 4 groups (6 rats/group) as follows: The control (saline), model (6-OHDA), mimic (6-OHDA + miR-335), or inhibitor (6-OHDA + miR-335 inhibitor). The control group was intracerebrally injected with saline, while the model group, the mimic group, and the inhibitor group received intracerebral injections of 6-OHDA. On post-surgery day 29, the mimic group and the inhibitor group received intracerebral injections of rno-miR-335 mimic (100 pmol/rat; micrON ${ }^{\mathrm{TM}}$ miRNA agomir; 5'-UCAAGA GCAAUAACGAAAAAUGU-3') or rno-miR-335 inhibitor (200 $\mathrm{pmol} / \mathrm{rat}$; micrOFF ${ }^{\mathrm{TM}}$ miRNA antagomir; 5'-ACA UUUUUCGUUAUUGCUCUUGA-3') into the right SNpc: Anteroposterior $(\mathrm{A} / \mathrm{P})=-4.9 \mathrm{~mm}$, mediolateral $(\mathrm{M} / \mathrm{L})=-1.9 \mathrm{~mm}$, dorsoventral $(\mathrm{D} / \mathrm{V})=-7.5 \mathrm{~mm})$. Both products were obtained from Guangzhou RiboBio Co., Ltd.

Behavioral test. On post-surgery days 0,28 and 30, the animals were subjected to the spontaneous rotation test. Apomorphine (APO, $0.5 \mathrm{mg} / \mathrm{kg}$; cat. no. 017-18321; Wako Pure Chemical Industries, Ltd.) was injected intraperitoneally. The rats were placed on a circular platform $(50 \mathrm{~cm}$ in diameter). Rotational speed was recorded for overall $30 \mathrm{~min}$. Rotations were counted manually $10 \mathrm{~min}$ after the APO injection. The striatal dopamine receptors can be super-sensitized due to a marked decrease in striatal dopamine concentration by 6-OHDA injection. Therefore, apomorphine, which is a dopamine receptor agonist, injected into the peripheral tissues, can induce the rats to exhibit rotational behavior to the left. A rotation was defined as a complete full-body turn $\left(360^{\circ}\right)$ towards the left side of 
the rat with one hind-paw as center and without switching head direction. If the rat turns to the right side, this did not count. Rats that turned $>7$ times/min were considered to have developed PD in this model $(31,32)$.

In vitro model of $P D$ and transfection. $\mathrm{PC} 12$ cells were purchased from ATCC (cat. no. CRL-1721) and cultured in RPMI-1640 medium (cat. no. C11875500BT; Gibco; Thermo Fisher Scientific, Inc.) containing $10 \%$ fetal bovine serum (cat. no. 10270-106; Gibco; Thermo Fisher Scientific, Inc.). The model group was established by stimulating the cells with 6-OHDA $(50 \mu \mathrm{M})$ for $24 \mathrm{~h}$. Erastin (cat. no. HY-15763, $1 \mu \mathrm{M}$ ), ferrostatin-1 (Fer-1; cat. no. HY-100579, $2.5 \mu \mathrm{M}$ ), and deferoxamine (DFO; cat. no. HY-B0988, $25 \mu \mathrm{M}$ ) were purchased from MedChemExpress. The cells were cultured in 6-well plates at $37^{\circ} \mathrm{C}$ in an incubator $\left(5 \% \mathrm{CO}_{2}\right)$. Cells in the logarithmic growth phase were transfected for $24 \mathrm{~h}$ with either negative control siRNA (siRNA NC), FTH1 siRNA (siRNA-FTH1-1, siRNA-FTH1-2, siRNA-FTH1-3; $100 \mathrm{nM}$ ), negative control miRNA inhibitor (inhibitor NC), negative control miRNA mimic (mimic NC), miR-335 inhibitor (inhibitor), or miR-335 mimic (mimic) (GenePharma, Inc.). Transfections were carried out using Lipofectamine 3000 (cat. no. L3000015, Thermo Fisher Scientific, Inc.) according to the manufacturer's guidelines. After $24 \mathrm{~h}$, western blot analysis was performed to select the most efficient siRNA-FTH1 sequence.

MTS assay. PC12 cells were inoculated in 96-well plates and 3 complex wells. Following induction with various concentrations of 6-OHDA $(0,10,50$ and $250 \mu \mathrm{M}), 20 \mu 1$ MTS solution (cat. no. G5421; Promega Corporation) mixed with $100 \mu \mathrm{l}$ RPMI-1640 medium was added to each well at 2 time points (12 and $24 \mathrm{~h}$ ). Cells were then transferred to a $5 \% \mathrm{CO}_{2}$ incubator at $37^{\circ} \mathrm{C}$ for $1 \mathrm{~h}$. An automated microplate reader was used to detect the absorbance $(490 \mathrm{~nm})$ to determine cell proliferation.

Candidate miRNA selection. The miRNA candidates potentially regulating FTH1 expression were selected from a list created by a bioinformatics search of three web-based algorithms: TargetScan (http://www.targetscan.org/), miRDB (http://www.mirdb.org/), and PicTar (http://www.pictar.org/). The parameters were set according to previous studies (33-35).

Total RNA extraction and RT-qPCR. For RT-qPCR, 12 rats were randomly classified into 2 groups (6 rats/group): The control (saline) and model (6-OHDA). Carbon dioxide asphyxiation was performed to sacrifice the animals. A total of 11 rats (one rat from the model group that did not reach the modeling standard was excluded from the experiment) were placed in a transparent and airtight chamber $(40 \times 20 \times 20 \mathrm{~cm})$ and gassed with carbon dioxide at a rate of $30 \%$ of the chamber volume per minute. The tissues were collected after the animal death was ensured by observing the movement, heartbeat and respiration. DNA-free RNA was obtained from SN tissue or PC12 cells using the Total RNA Extraction kit (cat. no. LS1040; Promega Corporation), and $500 \mathrm{ng}$ of total RNA were reverse transcribed using the PrimeScript RT reagent kit (cat. no. RR600A, Takara Bio, Inc.) according to the manufacturer's protocol, at $37^{\circ} \mathrm{C}$ for $15 \mathrm{mins}$ and $85^{\circ} \mathrm{C}$ for $30 \mathrm{sec}$. qPCR was performed in triplicate with TB Green Premix Ex Taq II (cat. no. RR820A, Takara Bio, Inc.) using a Light Cycler 480 SYBR-Green I Master Mix (Roche Diagnostics, GmbH). The amplification process was as follows: Denaturation at $95^{\circ} \mathrm{C}$ for $30 \mathrm{sec}$, followed by 40 cycles of denaturation at $95^{\circ} \mathrm{C}$ for $5 \mathrm{sec}$, annealing at $60^{\circ} \mathrm{C}$ for $30 \mathrm{sec}$ and extension at $50^{\circ} \mathrm{C}$ for $30 \mathrm{sec}$. The U6 gene was used for the normalization of miRNA expression. The primers for qPCR were as follows: miR-335 RT primer, 5'-GTCGTATCCAGTGCAGGGTCCGAGGTA TTCGCACTGATACGACACATTT-3'; forward, 5'-CGGCGC TCAAGAGCAATAACGAA-3' and reverse, 5'-ATCCAGTGC AGGGTCCGAGG-3'; U6 RT primer 5'-GTCGTATCCAGT GCAGGGTCCGAGGTATTCGCACTGGATACGACAAA ATA-3'; forward, 5' AGAGAAGATTAGCATGGCCCCTG-3' and reverse, 5'-ATCCAGTGCAGGGTCCGAGG-3'. Relative expression levels were analyzed using the $2^{-\Delta \Delta \mathrm{Cq}}$ method (36).

Western blot analysis. Cellular and SN tissue protein were incubated in RIPA buffer (cat. no. R0278; Sigma-Aldrich; Merck KGaA) for $1 \mathrm{~h}$, then centrifuged at 12,000 x $\mathrm{g}$ for $15 \mathrm{~min}$ at $4^{\circ} \mathrm{C}$. The extracted total proteins were eluted into EP tubes, and the concentration was measured using the bicinchoninic acid (BCA) assay (cat. no. 23225, Thermo Fisher Scientific, Inc.). Protein samples were separated on a $10 \%$ SDS-PAGE gel and transferred to polyvinylidene difluoride (PVDF) membranes (EMD Millipore). Membranes were blocked for $1 \mathrm{~h}$ at room temperature with $5 \%$ BSA with antibodies for tyrosine hydroxylase (TH) (1:1,000; cat. no. AB152, EMD Millipore), GPX4 (1:1,000; cat. no. ab125066, Abcam), FTH1 (1:1,000; cat. no. ab183781, Abcam) and $\beta$-tubulin $(1: 1,000$; cat. no. $2146 \mathrm{~S}$, Cell Signaling Technology, Inc.) overnight at $4^{\circ} \mathrm{C}$. The following day, the membranes were incubated in the presence of goat anti-rabbit secondary antibody $(1: 1,000$; cat. no. $7074 \mathrm{~S}$, Cell Signaling Technology, Inc.) for $2 \mathrm{~h}$ at room temperature. Bands were observed with Protein Simple FluorChem E. Data were analyzed using ImageJ software (version 1.52a; National Institutes of Health).

Immunohistochemistry (IHC). A total of 12 rats were randomly divided into 2 groups (6 rats/group): The control (saline) and model (6-OHDA). On post-surgery day 28, a total of 11 rats (1 rat from the model group that did not reach the modeling standard was excluded from the experiment) were anesthetized with $100 \mathrm{mg} / \mathrm{kg}$ ketamine and $10 \mathrm{mg} / \mathrm{kg}$ xylazine intraperitoneally. The cardiac aorta injection, blood washing and brain tissue fixation were performed using normal cold saline and $4 \%$ paraformaldehyde solution. Frozen sections ( $20-\mu$ m-thick) were subjected to antigen retrieval under high pressure using a heated citrate buffer. Immunohistochemical staining was performed using Histostain-Plus kits (cat. no. SP-0022, BIOSS) according to the manufacturer's protocol. Tissues were incubated in the presence of primary antibodies against TH (1:250; cat. no. AB152, EMD Millipore), GPX4 (1:250; cat. no. ab125066, Abcam) and FTH1 (1:250; cat. no. ab183781, Abcam) at $4^{\circ} \mathrm{C}$ overnight. After washing, the sections were incubated with secondary antibody (cat. no. ab6721; horseradish peroxidase-conjugated goat anti-rabbit; 1:5,000; Abcam). Then, diaminobenzidine (DAB) (cat. no. C02-04001; BIOSS) was added, and controlled color development was performed under a light microscope (Olympus, Japan) for strictly controlled amounts of time. Tissues were counter-stained 
with hematoxylin (cat. no. BA4097, BaSO Biotech) for $10 \mathrm{sec}$ at room temperature, dehydrated gradually with ethanol and coverslipped. Proteins of interest were quantified and analyzed using ImageJ software (version 1.52a; National Institutes of Health). The threshold feature in the menu of ImageJ software was used to count immune cells. Each immune cell was quantified using three IHC images, and the upper, middle, lower, left and right parts of each IHC image were selected.

Cellular iron concentration assay. The cellular iron concentration level was detected using a Quantichrom Iron Assay kit (cat. no. DIFE-250, BioAssay Systems). PC12 cells were cultured in 96-well plates and transfected with miR-335 mimic, miR-335 inhibitor, miR-335 NC, or siRNA-FTH1, and stimulated with 6-OHDA for $24 \mathrm{~h}$. Following the manufacturer's protocol, $50 \mu \mathrm{l}$ of standards or samples containing $10^{6}$ cells were mixed with $200 \mu \mathrm{l}$ Quantichrom Working Reagent in a 96-well plate and incubated at room temperature overnight. The optical density (OD) was measured at a wavelength of $590 \mathrm{~nm}$ using a microplate reader (BioTek Instruments, Inc.).

MMP assay. MMP was detected by JC-1 staining (Beijing Solarbio Science \& Technology Co., Ltd.). Briefly, the PC12 cells were cultured in 6-well plates, then washed with PBS. Cells were incubated with JC-1 stain $(0.5 \mathrm{ml})$ at $37^{\circ} \mathrm{C}$ for $20 \mathrm{~min}$. A fluorescence microscope (Olympus Corporation) was used to visualize MMP.

Intracellular ferrous ion imaging. Intracellular ferrous ion distribution was assessed using a FeRhoNox-1 staining kit (Goryo Chemical, Inc.). PC12 cells $\left(5 \times 10^{4} /\right.$ well) were seeded in a 24-well microplate overnight. Following transfection with miR-335 mimic, miR-335 inhibitor, miR-335 NC, or siRNA-FTH1, and stimulation with 6-OHDA $(50 \mu \mathrm{M})$ for $24 \mathrm{~h}$, the cell culture medium was removed. The cells were then incubated with $1 \mu \mathrm{M}$ FeRhoNox- 1 at $37^{\circ} \mathrm{C}$ for $1 \mathrm{~h}$. After washing with PBS 3 times, $\mathrm{Fe}^{2+}$ distribution was imaged using a Cytation $5^{\mathrm{TM}}$ Cell Imaging Multi-Mode Reader (BioTek Instruments, Inc.).

Lipidperoxidationgenerationdetection. $\mathrm{PC} 12$ cells $\left(1 \times 10^{5} /\right.$ well) were seeded in 6-well plates overnight, and the cells were treated as follows: PC12 cells were transfected with miR-335 mimic, miR-335 inhibitor, miR-335 NC, or siRNA-FTH1, respectively, and then stimulated with 6-OHDA $(50 \mu \mathrm{M})$ for 24 h; PC12 cells were treated with Erastin (cat. no. HY-15763, MedChem Express), Fer-1 (cat. no. HY-100579, MedChem Express), DFO (cat. no. HY-B0988, MedChem Express) or Z-VAD-FMK (cat. no. HY-16658B MedChem Express) for $24 \mathrm{~h}$, respectively, and then stimulated with 6-OHDA $(50 \mu \mathrm{M})$ for $24 \mathrm{~h}$. The cells were incubated with $10 \mu \mathrm{M}$ C11-BODIPY (581/591; cat. no. D3861, Thermo Fisher Scientific, Inc.) for $30 \mathrm{~min}$ at $37^{\circ} \mathrm{C}$, then washed with PBS 3 times. Cells were harvested with trypsin (cat. no. 15050-065, Gibco; Thermo Fisher Scientific, Inc.), suspended in fresh medium, and analyzed using a CytoFLEX flow cytometer (Beckman Coulter, Inc.). Fluorescence was measured by acquiring green $(484 / 510 \mathrm{~nm})$. The butadienyl portion of C11-BODIPY581/591 was oxidized, resulting in the $510 \mathrm{~nm}$ fluorescence emission peak. The data were analyzed using FlowJo v10 software.
Cellular ROS detection. Cells were cultured in 6-well plates, and then collected into tubes. Buffer (10X, provided with the kit) and $\mathrm{ddH}_{2} \mathrm{O}$ were mixed at a ratio of $1: 9$ gently and thoroughly to yield $1 \mathrm{X}$ buffer. Cells were resuspended with $1 \mathrm{ml} 1 \mathrm{x}$ buffer and were stained with $25 \mu \mathrm{M}$ DCFDA (cat. no. ab113851; Abcam) in the dark at $37^{\circ} \mathrm{C}$ for $30 \mathrm{~min}$, then analyzed using a CytoFLEX flow cytometer (Beckman Coulter, Inc.; $535 \mathrm{~nm}$ ).

Dual luciferase reporter system assay. Dual luciferase reporter (DLR) experiments were performed on the PC12 cells. miR-335 and negative control mimic were purchased from GenePharma Co., Ltd. First, pLUc-FTH1-wild-type (WT) and pLUc-FTH1-mutant (MUT) plasmids (cat. nos. U6885-02 and U6886-02, respectively; Shenzhen Huaan Ping Kang Bio Technology, Inc.) were constructed. Cells were transfected using either WT (200 ng) or MUT (200 ng) constructs, with the miR-335 inhibitor $(100 \mathrm{nM})$, miR-335 mimic (100 $\mathrm{nM})$, mimic negative control $(100 \mathrm{nM})$ or inhibitor negative control $(100 \mathrm{nM})$. At $24 \mathrm{~h}$ following transfection, the DLR assay kit (E2920; Promega Corporation) was utilized to detect luminescence, according to the manufacturer's protocol. The absorbance values of sea cucumber luciferase served as an internal reference to perform normalized statistical calculations.

Statistical analysis. All experiments were carried out 3 times. Data analysis was performed using GraphPad Prism 8 (GraphPad Software, Inc.). Student's t-tests were used to determine the difference between the 2 groups for the behavioral data, immunohistochemistry, RT-qPCR, MTS assay and western blot analysis data (PC12 cells induced with gradient 6-OHDA and transfected with FTH1 siRNA). One-way ANOVA followed by post hoc Tukey's multiple comparison tests were used to analyze the difference between groups in the remaining western blot analysis data, cellular iron concentration assays, MMP, cell ROS detection and DLR assay data. Data are presented as the means \pm SEM. $\mathrm{P}<0.05$ was considered to indicate a statistically significant difference.

\section{Results}

TH, GPX4 and FTH1 expression levels are downregulated following 6-OHDA induction in rats. The successful establishment of the rat model of PD was assessed by performing behavioral testing on post-surgery day 28 . A total of 28 rats demonstrated $>7$ turns/min, and 2 rats that did not reach the modeling standard were not included in the subsequent experiments $(\mathrm{P}<0.0001$; Fig. 1A). Brain tissue was harvested, and immunohistochemistry was performed to detect $\mathrm{PD}$-associated pathology and ferroptosis. TH is a protein marker of dopaminergic neurons in the nervous system (37). On day 28 , the model rats exhibited a markedly reduced $\mathrm{TH}$ expression in the $\mathrm{SN}$, demonstrating a significant loss of dopaminergic neurons ( $\mathrm{P}=0.0125$; Fig. 1B). GPX4 is a vital marker protein of ferroptosis. The results revealed that rats exposed to 6-OHDA exhibited a reduced expression of GPX4 and FTH1, demonstrating that FTH1 expression was negatively associated with the level of ferroptosis and revealing a positive association between ferroptosis and PD in the model rats 


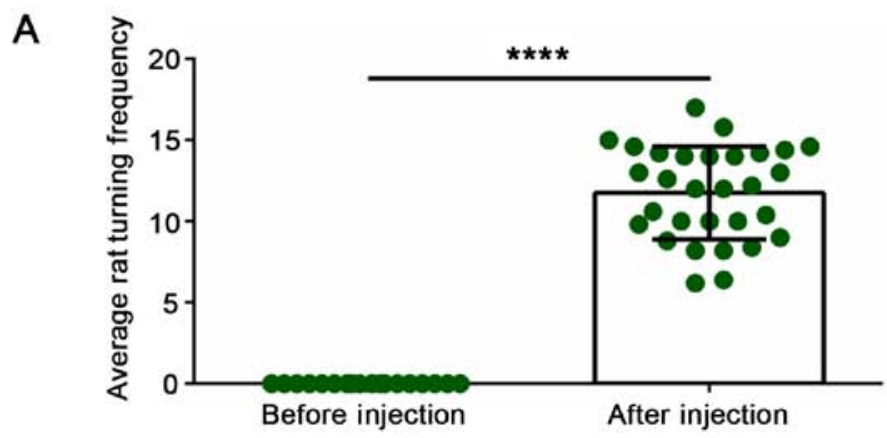

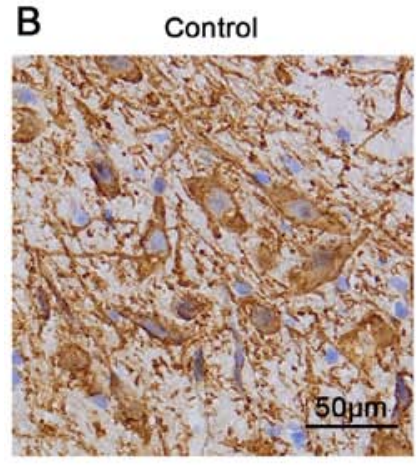

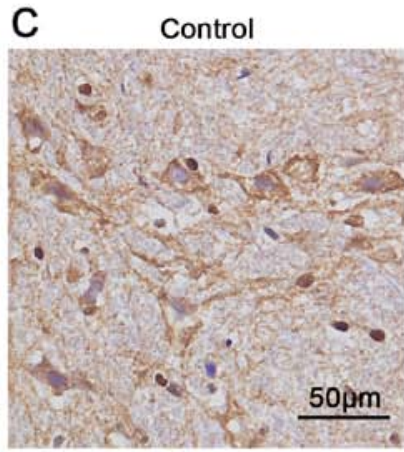

D

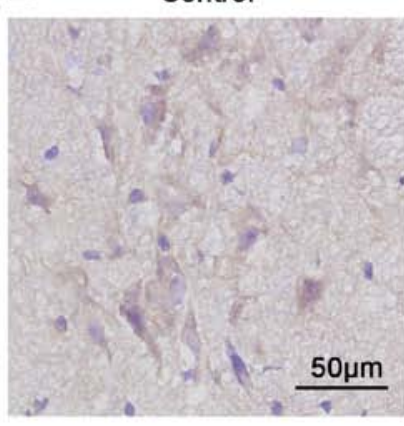

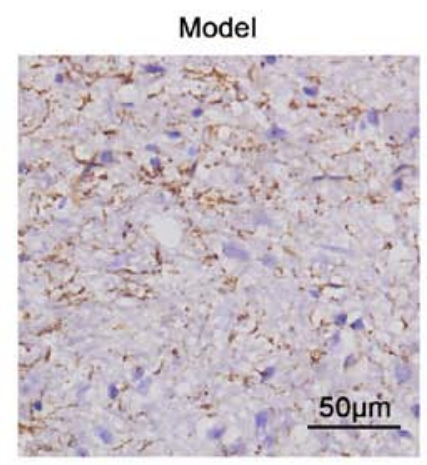

Model

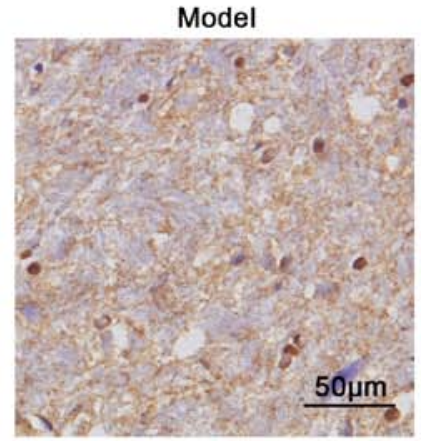

Model

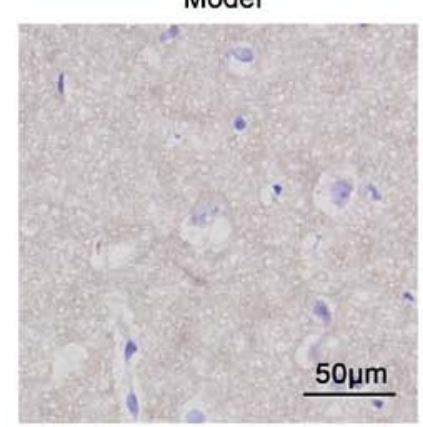

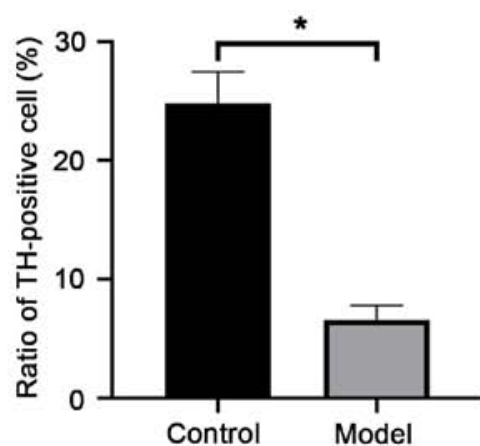
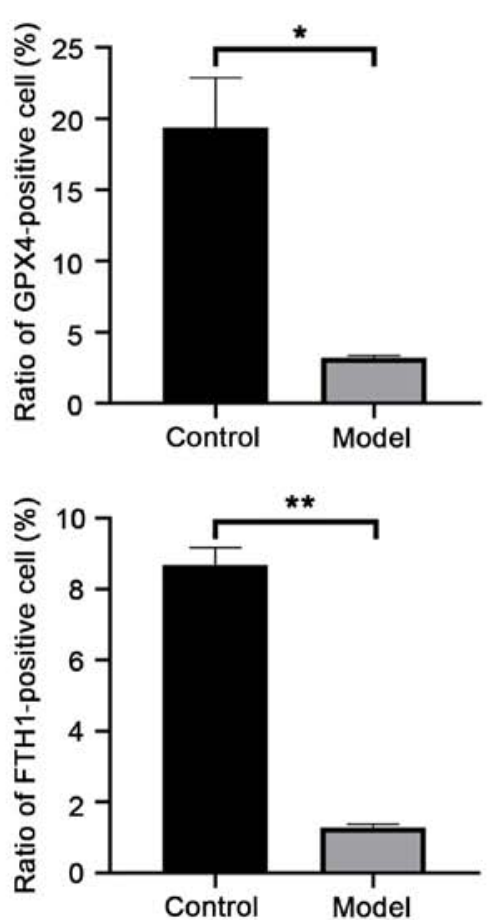

Figure 1. The expression levels of TH, GPX4 and FTH1 are significantly decreased in rats with PD. (A) The change in the number of rotation laps of the rats after the apomorphine (APO) injection on post-surgical day 28 ; the number of turns is presented as the mean \pm SEM. (B) IHC was performed in the SN of the control and model group. The expression of TH was decreased in the model group. (C) The expression of GPX4 was decreased in the model group. (D) The expression of FTH1 was reduced in the model group. The ratio of positive cells was analyzed. ${ }^{*} \mathrm{P}<0.05,{ }^{* * *} \mathrm{P}<0.01$ and ${ }^{* * * * *} \mathrm{P}<0.0001$ vs. model group; scale bar, $50 \mu \mathrm{m}$. PD, Parkinson's disease; TH, tyrosine hydroxylase; GPX4, glutathione peroxidase 4; FTH1, ferritin heavy chain 1.

(GPX4, P=0.0229; FTH1, P=0.0023; Fig. 1C and D). Therefore, it is useful to explore the upstream molecules that may target FTH1 to regulate ferroptosis in PD.

miR-335 is upregulated in PD and promotes ferroptosis and PD pathology. The target miRNAs of FTH1 were predicted using three prediction algorithms online: TargetScan (http://www. targetscan.org/), miRDB (http://www.mirdb.org/), and PicTar (http://www.pictar.org/). The parameters were set according to previous studies (35-37). The differential expression of all miRNAs predicted by 3 prediction algorithms in the the SN of control and model rats were validated by RT-qPCR. The expression of miR-335 was significantly higher in the model rats $(\mathrm{P}<0.0001$; Fig. $2 \mathrm{~A})$. To verify the damaging effects of miR-335 in the model rats, the rats were infected with either miR-335 mimic or miR-335 inhibitor on day 29. The results 

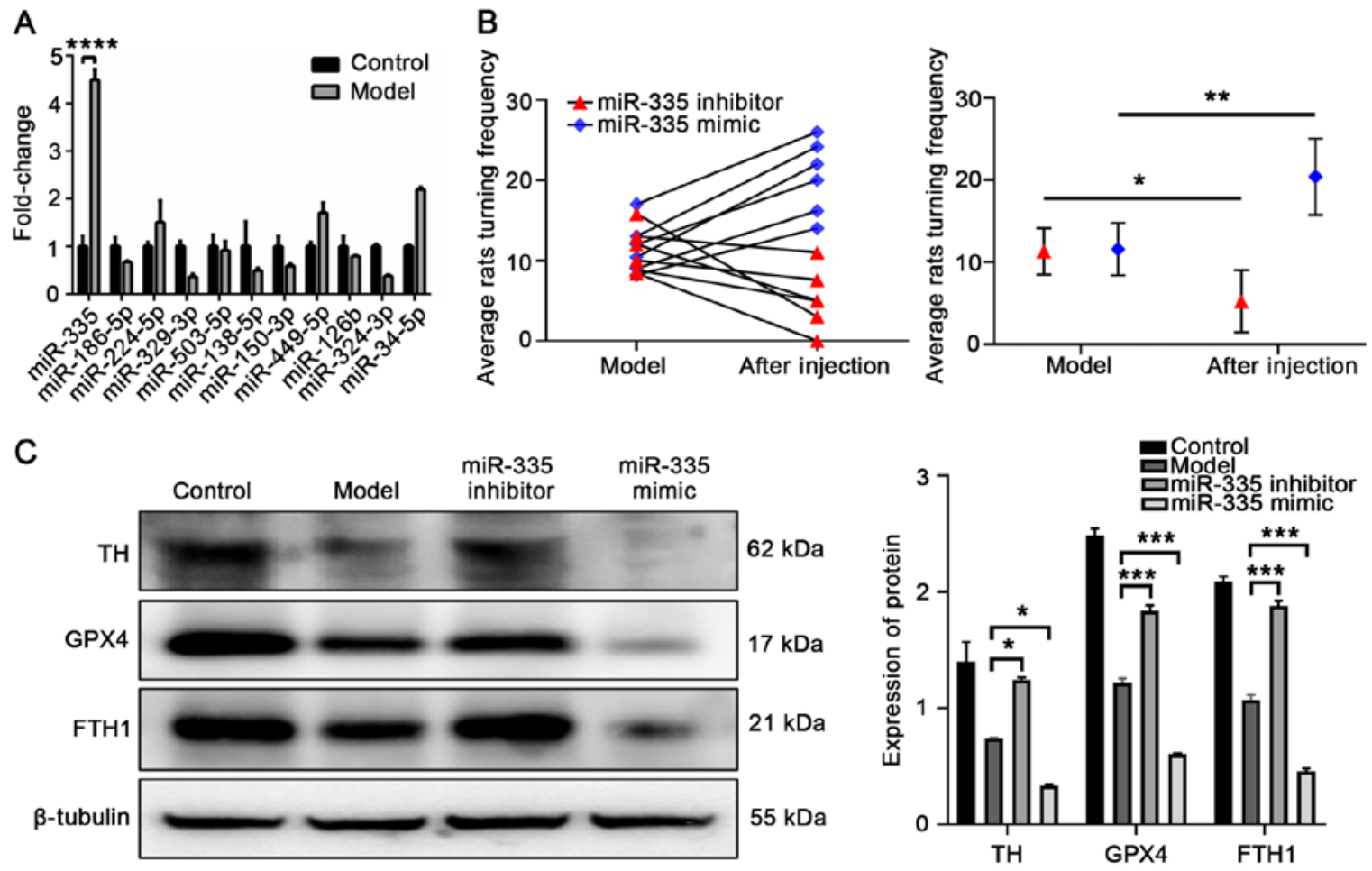

Figure 2. miR-335 promotes ferroptosis in rats with PD. (A) The expression of miR-335 was significantly increased in rats with PD. RT-qPCR was performed to select the most differentially expressed miRNA in the $\mathrm{SN}$ of rats. ${ }^{*} \mathrm{P}<0.05$ vs. model group. (B) miR-335 significantly increased the number of rotation laps in rats with PD. The graph on the right panel shows the results of the statistical analysis of findings on the graph on the left panel. miR-335 inhibitor vs. model, ${ }^{*} \mathrm{P}<0.05$. miR-335 mimic vs. model, ${ }^{* *} \mathrm{P}<0.01$. (C) miR-335 mimic reduced $\mathrm{TH}, \mathrm{GPX} 4$ and FTH1 expression levels in rats with $\mathrm{PD}$. ${ }^{*} \mathrm{P}<0.05$, ${ }^{* *} \mathrm{P}<0.01$ and ${ }_{* * * *} \mathrm{P}<0.001$ vs. model group. PD, Parkinson's disease; TH, tyrosine hydroxylase; GPX4, glutathione peroxidase 4; FTH1, ferritin heavy chain 1.

of the behavioral analysis revealed that the rotations in the mimic group significantly increased $(\mathrm{P}=0.0033$ compared to the model group; Fig. 2B), while those in the inhibitor group significantly decreased $(\mathrm{P}=0.0103$ compared to the model group; Fig. 2B). These results indicated that miR-335 exacerbated the behavioral deficits in rats with PD. Western blot analysis was then used to detect the effects of miR-335 on the degree of PD pathology and ferroptosis in the SN of rats. miR-335 mimic significantly exacerbated PD injury and promoted ferroptosis compared to the model group by decreasing TH and GPX4 expression (TH, P=0.0329; GPX4, $\mathrm{P}=0.0005$; Fig. 2C), and FTH1 expression was also markedly downregulated ( $\mathrm{P}=0.0004$; Fig. $2 \mathrm{C}$ ). These results indicated that miR-335 promoted PD pathology and ferroptosis, and decreased FTH1 protein expression in PD rats. Moreover, there was a negative association between miR-335 and FTH1, suggesting that miR-335 may negatively regulate the expression of FTH1 in vivo.

miR-335 targets the 3'UTR of FTH1 mRNA. Based on these results, miR-335 was predicted to regulate FTH1. The present study then investigated whether miR-335 functions by binding with the 3'UTR of FTH1 mRNA. The binding site between miR-335 and FTH1 was predicted using TargetScan, and the plasmid profile is illustrated in Fig. 3A. A DLR assay was then conducted to demonstrate their interaction and determined an inhibitory effect of miR-335 mimic on the luciferase activity of WT FTH1 (FTH1 WT) after $24 \mathrm{~h}$ of transfection. Compared with the miR-335 mimic NC + FTH1 WT group, the relative luciferase activity of the miR-335 mimic + FTH1 WT group was significantly decreased $(\mathrm{P}=0.0088$, Fig. 3B); compared with the miR-335 inhibitor NC + FTH1 WT group, the relative luciferase activity of the miR-335 inhibitor + FTH1 WT group was significantly increased (Fig. 3B, $\mathrm{P}=0.0387$ ); there was no statistically significant difference between each group, following co-transfection with the FTH1-MUT plasmid (Fig. 3B, $\mathrm{P}<0.05$ ). These results demonstrate that miR-335 targets the 3'UTR of FTH1 mRNA.

Expression of TH, GPX4 and FTH1 is decreased in 6-OHDA-stimulated cells. Cell activity assays were performed to explore the optimal 6-OHDA induction time and concentration to model PD in vitro. As shown in Fig. 4A, $\mathrm{PC} 12$ cells were exposed to 6-OHDA at a range of concentrations $(0-250 \mu \mathrm{M})$ over a period of time $(12-24 \mathrm{~h})$. Cell viability was found to be reduced in a concentration- and time-dependent manner. The transient exposure of the PC12 cells to $50 \mu \mathrm{M}$ of 6 -OHDA for $24 \mathrm{~h}$ led to a $50 \%$ decrease in cell viability $(\mathrm{P}<0.0001)$. Thus, this protocol was adopted for use in subsequent experiments.

The present study then investigated whether the expression patterns of GPX4 and FTH1 were similar in the cell model to those of the rats with PD. Western blot analysis was used to detect the expression of TH, GPX4 and FTH1 in PC12 cells following stimulation with 6-OHDA. With the increasing 6-OHDA concentration, the expression of TH, GPX4 and FTH1 gradually decreased (Fig. 4B). These results demonstrated that with the onset of injury, the expression of $\mathrm{TH}$, GPX4 and FTH1 was downregulated, which corresponds to the results obtained in the animal experiments. 


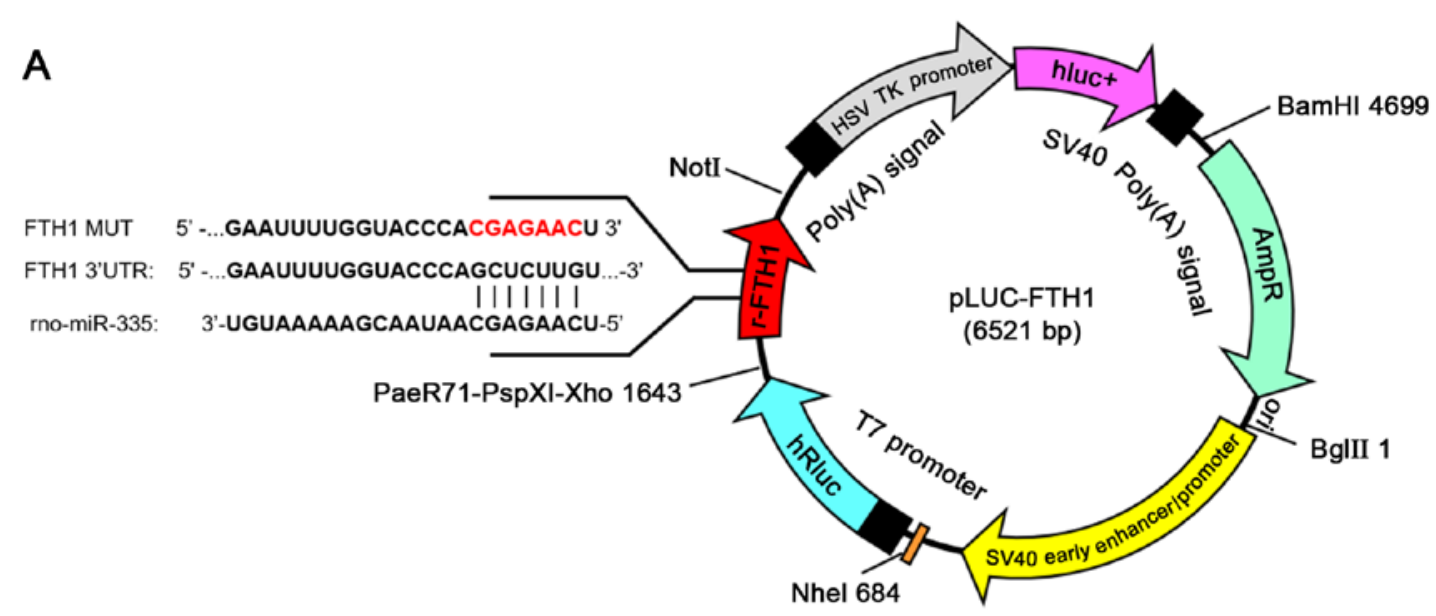

B
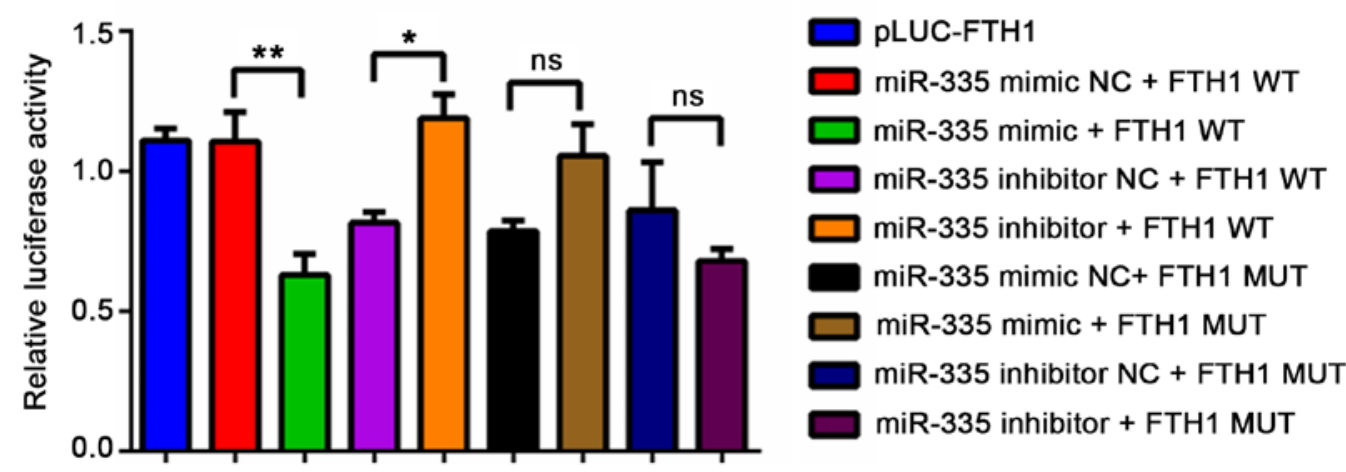

Figure 3. miR-335 targets the 3'-UTR of FTH1 specifically. (A) TargetScan and miRBase were used to investigate the binding site of miR-335 and the 3'-UTR of FTH1. The construction profile of the pLUC-FTH1 plasmid is presented. The binding sequence of 3'UTR of FTH1 was replaced by the pLUC-FTH1 MUT plasmid. (B) Luciferase activity of miR-335 inhibitor (100 nM), miR-335 mimic (100 nM), miR-335 inhibitor negative control (100 nM) or miR-335 mimic negative control $(100 \mathrm{nM})$ in the presence of the FTH1 WT (200 ng) or FTH1 MUT (200 ng) was detected using the dual luciferase reporter assay. ${ }^{*}$ P $<0.05 \mathrm{vs.} \mathrm{miR-335}$ inhibitor NC + FTH1 WT group; ${ }^{* *}$ P<0.01 vs. miR-335 mimic NC + FTH1 WT group. FTH1, ferritin heavy chain 1; WT, wild-type; MUT, mutated type.

Subsequently, the effect sof inducer or inhibitor of ferroptosis and inhibitors of other cell death mechanisms (such as apoptosis) on 6-OHDA-stimulated cells were investigated. The effects of the ferroptosis inducer, erastin, and the inhibitors, Fer-1 and DFO, on the expression of TH, GPX4 and FTH1 were investigated in 6-OHDA-stimulated cells. As shown in Fig. 4C, erastin treatment aggravated the effects of 6-OHDA. Compared with the model group, the expression of TH, GPX4 and FTH1 was significantly downregulated in the erastin group (cells stimulated with 6-OHDA and erastin). These effects were attenuated by the ferroptosis inhibitor, Fer-1, and the iron chelator, DFO (38). The level of lipid peroxides was increased by $21.9 \%$ in the erastin group $(\mathrm{P}=0.0015)$ and decreased by $35.9 \%(\mathrm{P}<0.0001)$ and $44.4 \%(\mathrm{P}<0.0001)$ in the Fer-1 group and DFO group compared with model group (Fig. 4D). Moreover, the level of lipid peroxidation, and the expression levels of $\mathrm{TH}$, GPX4 and FTH1 were not affected by the apoptosis inhibitor, Z-VAD-FMK, compared with the model group (Fig. S1; $\mathrm{P}>0.05$ ). These results suggested that 6-OHDA induced some degree of ferroptotic death and ferroptosis was the main cell death mechanism of the 6-OHDA-induced cell models. FTH1 degradation accompanied the process of ferroptosis.

Taken together, the in vivo (Figs. 1 and 2) and in vitro (Fig. 4) results indicated that FTH1 expression was negatively associated with ferroptosis, and that ferroptosis was positively associated with PD pathology.
miR-335 increases ferroptosis and aggravates PD injury in 6-OHDA-stimulated cells. To determine the expression of miR-335 in 6-OHDA-stimulated cells, RT-qPCR was performed in 6-OHDA-stimulated cells. The results revealed that miR-335 was highly expressed in 6-OHDA-stimulated cells $(\mathrm{P}<0.0001$; Fig. 5A). The present study then determined whether miR-335 promotes ferroptosis in $\mathrm{PD}$ in vitro. The 6-OHDA-stimulated cells were transfected with miR-335. The inhibition of GPX4 function leads to the $\mathrm{Fe}^{2+}$-dependent formation of toxic lipid ROS, resulting in the induction of ferroptosis (8). Therefore, the expression of GPX4, the level of $\mathrm{Fe}^{2+}$, lipid peroxidation, cellular ROS and MMP were measured as indicators of ferroptosis. Western blot analysis was performed to detect the regulatory effects of miR-335 on GPX4, FTH1 and PD pathology in 6-OHDA-stimulated cells. As shown in Fig. 5B, miR-335 mimic significantly exacerbated PD pathology, and reduced GPX4 and FTH1 expression compared with the model group $(\mathrm{TH}, \mathrm{P}=0.1439$; GPX4, $\mathrm{P}=0.0322$; FTH1, $\mathrm{P}=0.0002$ ). The cellular iron concentration results revealed that $\mathrm{Fe}^{2+}$ increased by $21.1 \%$ in the mimic group compared with the model group ( $\mathrm{P}=0.0413$, Fig. 5C). In addition, the results of ferrous ion imaging confirmed that miR-335 further led to the accumulation of $\mathrm{Fe}^{2+}$ in 6-OHDA-stimulated cells (Fig. S2). Moreover, compared with the model group, both lipid peroxidation and the ROS level increased significantly in the mimic group $(\mathrm{P}=0.0312$, Fig. $5 \mathrm{D} ; \mathrm{P}<0.0001$, 

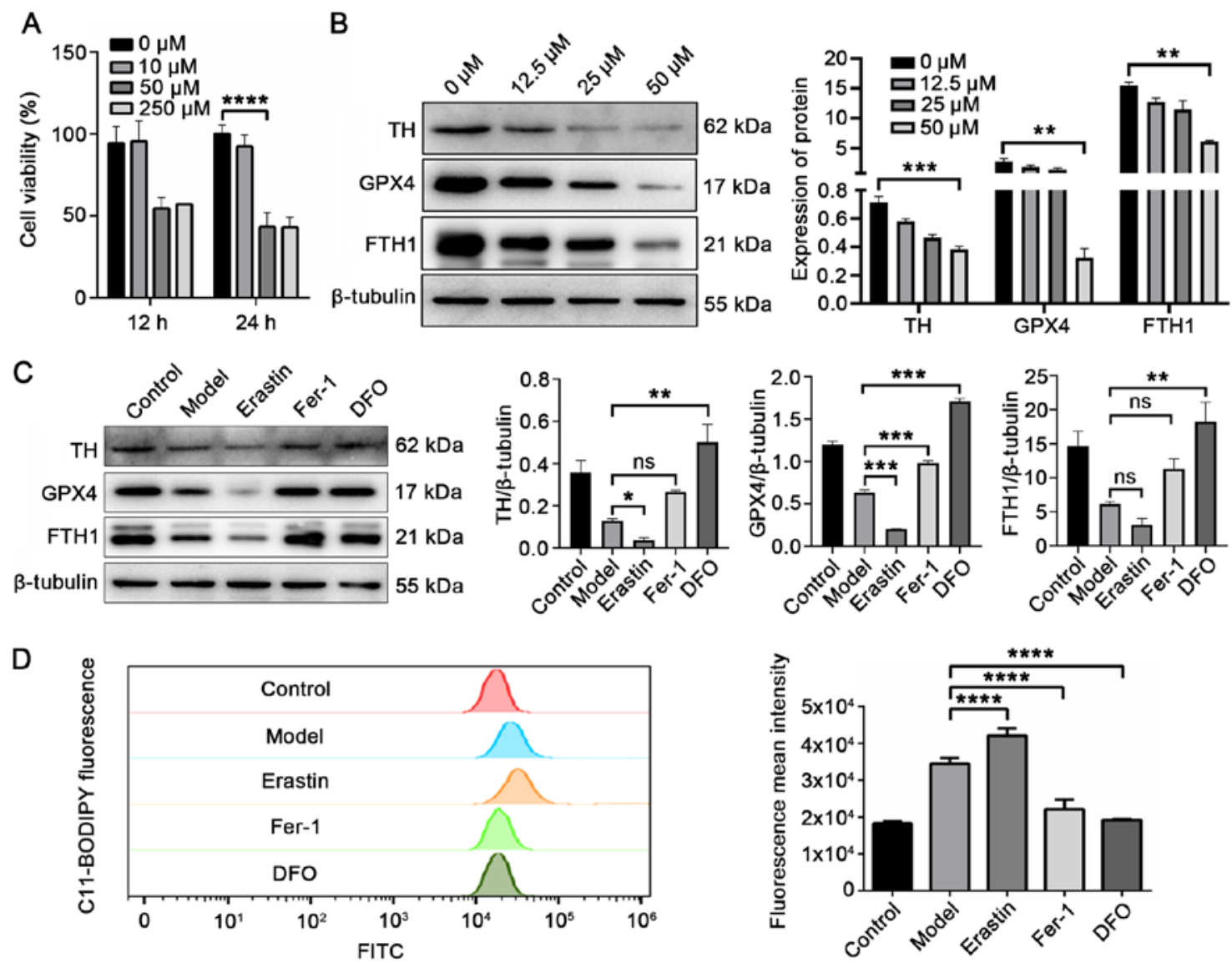

Figure 4. FTH1 expression is negatively associated with ferroptosis, and ferroptosis is positively associated with PD pathology. (A) Cell viability was significantly reduced to approximately 50\% following induction with $50 \mu \mathrm{M} 6$-OHDA for $24 \mathrm{~h}$. (B) Expression of TH, GPX4 and FTH1 was inversely proportional to 6-OHDA from 0 to $50 \mu \mathrm{M}$. (C) 6-OHDA-stimulted cells, further treated with the ferroptosis inducer, erastin (1 $\mu \mathrm{M})$, exhibited a reduced expression of TH, GPX4 and FTH1. The ferroptosis inhibitors, Fer-1 $(2.5 \mu \mathrm{M})$ and DFO $(25 \mu \mathrm{M})$, increased these expression levels. (D) Fluorescent C11-BODIPY staining and FACS analysis were used to evaluate the formation of lipid peroxides in model cells and 6-OHDA-stimulted cells co-treated with erastin (ferroptosis inducer), Fer-1 (ferroptosis inhibitor) and DFO (iron chelator). ${ }^{*} \mathrm{P}<0.05,{ }^{* *} \mathrm{P}<0.01,{ }^{* * *} \mathrm{P}<0.001$ and ${ }^{* * * *} \mathrm{P}<0.0001$ vs. model group; ns, not significant. PD, Parkinson's disease; FTH1, ferritin heavy chain 1; 6-OHDA, 6-hydroxydopamine; Fer-1, ferrostatin-1; DFO, deferoxamine.

Fig. 5E). Mitochondrial dysfunction is an important indicator of ferroptosis. Further detection of mitochondrial dysfunction was performed by JC-1 staining. When the MMP was high, JC-1 accumulated in the mitochondria matrix and formed a polymer; when MMP was low, it formed a monomer. As shown in Fig. 5F, MMP was significantly reduced in the mimic group $(\mathrm{P}=0.0426)$. These results indicated that $\mathrm{miR}-335$ inhibited the expression of GPX4 and FTH1, thereby increasing the level of $\mathrm{Fe}^{2+}$. The $\mathrm{Fe}^{2+}$-dependent accumulation of lipid ROS led to the mitochondrial dysfunction, which downregulated the level of MMP, resulting in ferroptosis and PD aggravation.

FTH1 silencing increases ferroptosis and further exacerbates $P D$ pathology. To further verify the role of FTH1 in PD pathology and ferroptosis in vitro, RNA interference (RNAi) was performed in 6-OHDA-stimulated cells to silence the expression of FTH1. siRNA-FTH1-1 was the most efficient sequence for silencing (Fig. 6A). Following transfection, the model and siRNA-FTH1 groups were stimulated with 6-OHDA. As shown in Fig. 6B, the TH and GPX4 expression levels were further decreased in the FTH1-siRNA-transfected 6-OHDA-stimulated cells $(\mathrm{P}<0.0001 ; \mathrm{P}=0.0071$ vs. model group). The analysis of the cellular iron concentration revealed that the $\mathrm{Fe}^{2+}$ concentration was increased by si-FTH1 ( $<<0.0001$; Fig. 6C). The ferrous ion imaging results also confirmed that si-FTH1 led to the further accumulation of $\mathrm{Fe}^{2+}$ in the 6-OHDA-stimulated cells (Fig. S3). Moreover, lipid peroxidation and cellular ROS levels were increased by si-FTH1 compared with the model group ( $\mathrm{P}=0.0061$, Fig. 6D; $\mathrm{P}<0.0001$, Fig. 6E). JC-1 staining indicated that MMP was decreased by si-FTH1 compared with the model group $(\mathrm{P}=0.0227$, Fig. $6 \mathrm{~F})$. These data provide further evidence that FTH1 plays a crucial role in PD pathology and ferroptosis. FTH1 degradation released a large amount of $\mathrm{Fe}^{2+}$, which led to the accumulation of toxic lipid peroxides and ROS to damage the mitochondria. The decline in the MMP level was the manifestation of mitochondrial dysfunction.

\section{Discussion}

The present study used 6-OHDA-induced rat and cell models to simulate the pathology of mid-late stage $\mathrm{PD}$, in order to explore the role of miRNA in the $\mathrm{Fe}^{2+}$ regulation of ferroptosis. The major findings were the following: i) In both the rat and cell models of PD, FTH1 expression was negatively associated with ferroptosis, and there was a positive association between ferroptosis and PD pathology; ii) miR-335 expression was increased in the rat model of PD, and targeted the 3'UTR of FTH1 mRNA; iii) the exacerbation of ferroptosis, promoted 
A

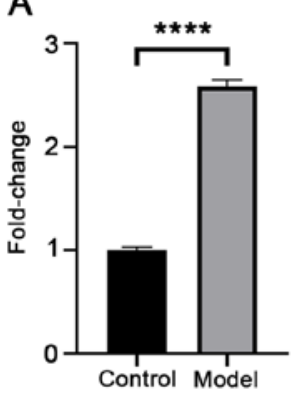

C

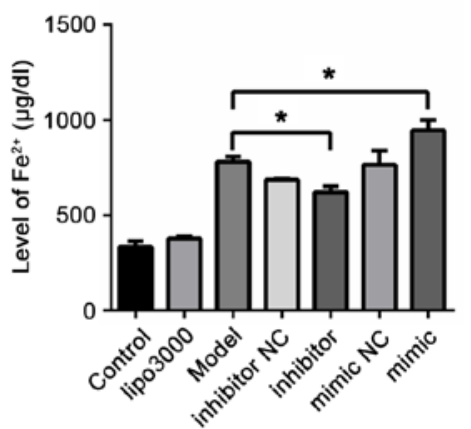

B

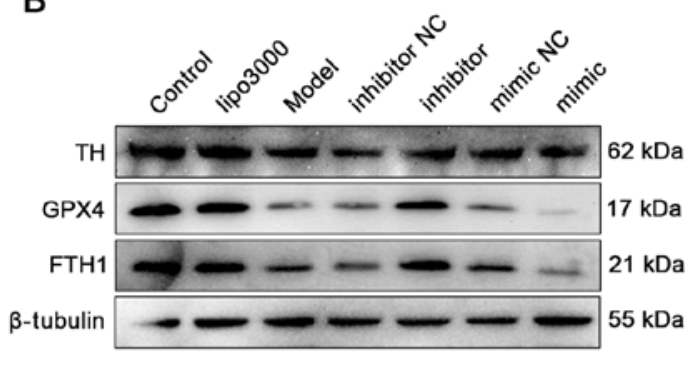

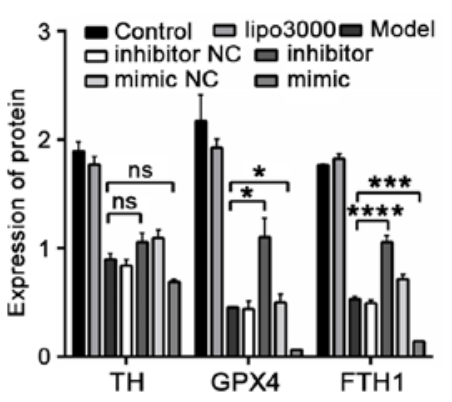

E

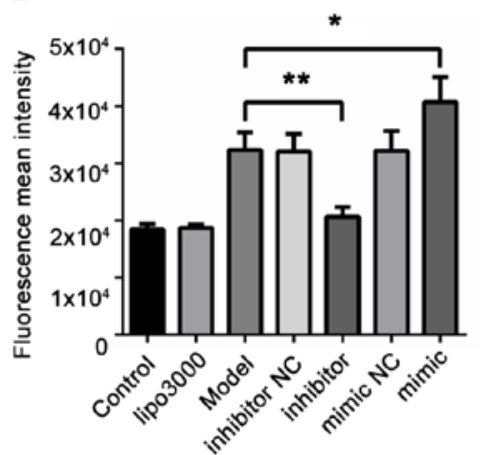

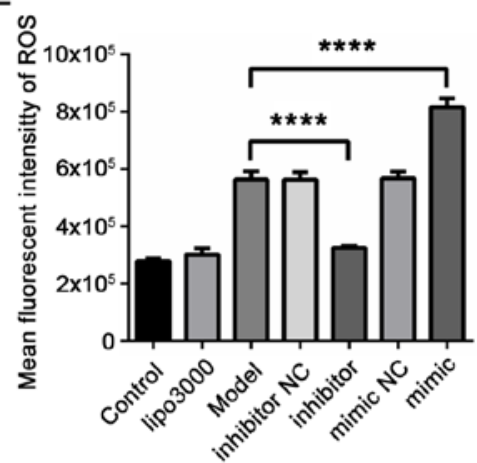

$\mathrm{F}$
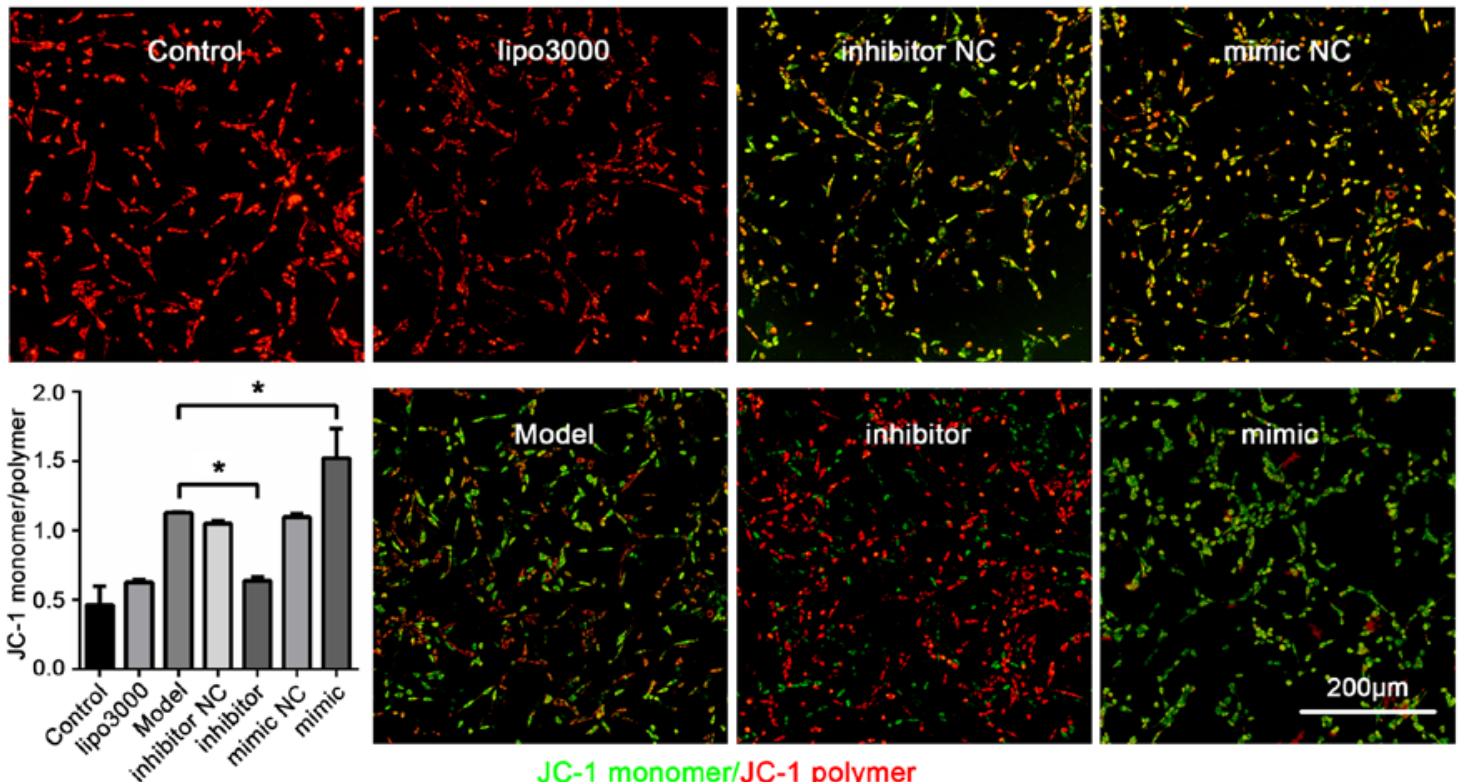

JC-1 monomer/JC-1 polymer

Figure 5. miR-335 promotes ferroptosis and reduces the expression of TH, GPX4 and FTH1 in 6-OHDA-stimulated cells. (A) Expression of miR-335 in 6-OHDA induced cells was markedly increased. (B) Expression of TH, GPX4 and FTH1 in PD cells was reduced by miR-335 mimic. (C) miR-335 increased the iron concentration in 6-OHDA-stimulated cells. (D) miR-335 increased lipid peroxidation in 6-OHDA-stimulated cells. (E) miR-335 significantly increased ROS levels in 6-OHDA-stimulated cells. (F) miR-335 downregulated MMP in 6-OHDA-stimulated cells. ${ }^{*} \mathrm{P}<0.05,{ }^{* * *} \mathrm{P}<0.01,{ }^{* * * *} \mathrm{P}<0.001$ and ${ }^{* * * * *} \mathrm{P}<0.0001$ vs. model group; ns, not significant. Scale bar, $200 \mu \mathrm{m}$. Parkinson's disease; TH, tyrosine hydroxylase; GPX4, glutathione peroxidase 4; FTH1, ferritin heavy chain 1; 6-OHDA, 6-hydroxydopamine.

by miR-335, increased the $\mathrm{Fe}^{2+}$ concentration by inhibiting FTH1 expression in PD. The results also identified a novel role of miR-335 to directly target FTH1, acting as an essential regulator for the ferroptosis signaling pathway in PD.

One of the pivotal results of the present study was that FTH1 expression was negatively associated with the level of ferroptosis in PD. In the present study, GPX4 and TH expression was decreased in the model group compared with the control group. The expression of TH, GPX4 and FTH1 in the 6-OHDA-stimulated cells was not affected by the apoptosis inhibitor, Z-VAD-FMK (Fig. S1A). The level of ferroptosis and PD pathology was increased using siRNA against FTH1, indicating that decreasing FTH1 expression can promote ferroptosis and dopaminergic neuron loss in PD. In a previous study, the authors demonstrated that FTH1 linked ferritinophagy to ferroptosis in a model of 6-OHDA-induced PD (14). The function of FTH1 is mainly to store $\mathrm{Fe}^{2+}$ in eukaryotic cells in a soluble and non-toxic form, thereby preventing ferroptosis $(39,40)$. The results of the present study are in accordance with those of previous studies identifying a protective role for FTH1 in $\mathrm{Fe}^{2+}$ storage and ferroptosis in PD $(13,15)$. Moreover, GPX4 expression was significantly reduced in neurons of the 
A

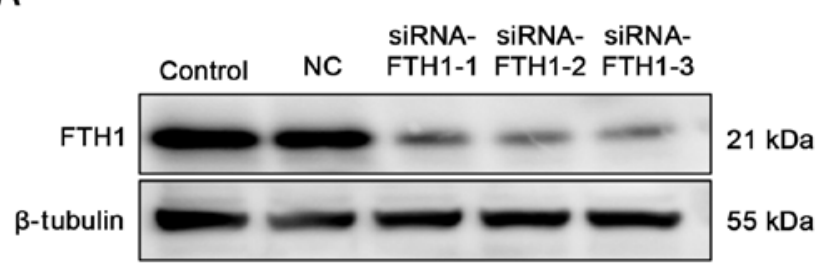

B

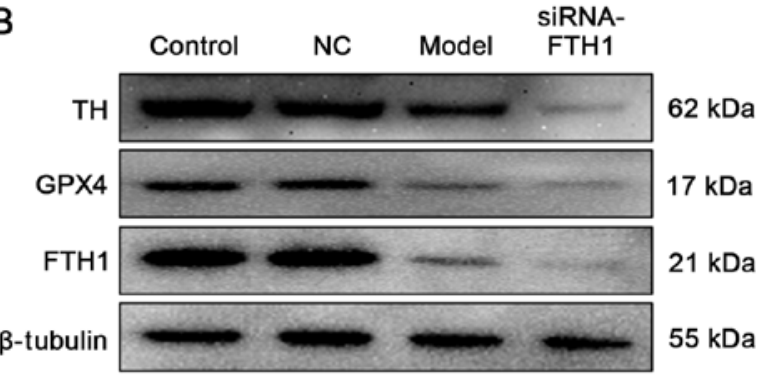

C

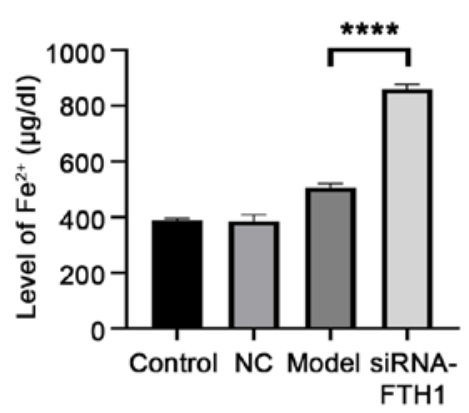

$\mathrm{F}$

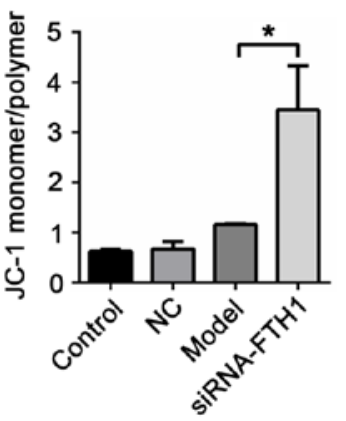

D

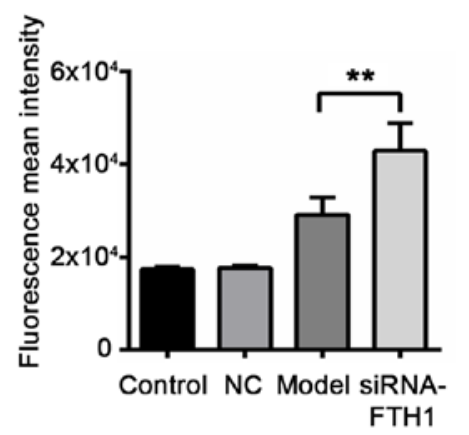

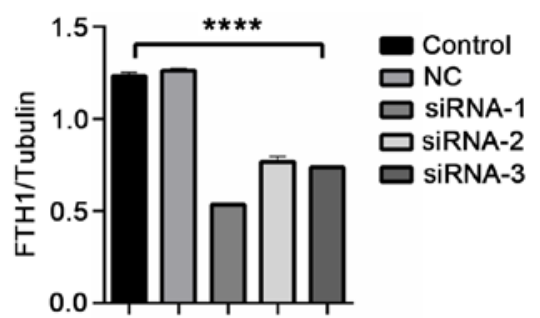

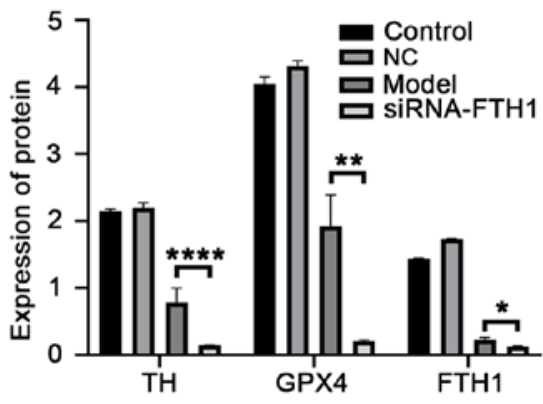

E

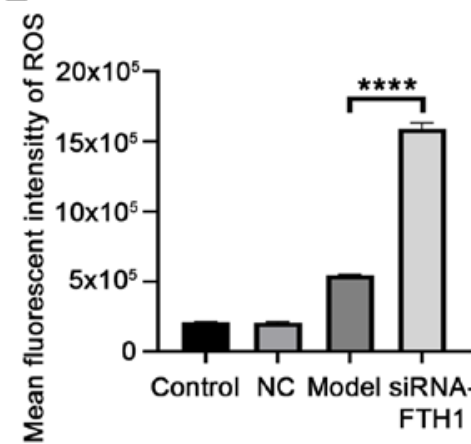

Figure 6. FTH1 silencing promotes ferroptosis and further decreases the expression of TH and GPX4. (A) siRNA-FTH1 (100 nM) transfection for $24 \mathrm{~h}$ efficiently reduced FTH1 expression. (B) FTH1 silencing reduced TH, GPX4, and FTH1 expression in 6-OHDA-stimulated cells. (C) FTH1 silencing increased the iron concentration in 6-OHDA-stimulated cells. (D) FTH1 silencing increased lipid peroxidation in 6-OHDA-stimulated cells. (E) FTH1 silencing promoted the accumulation of ROS in cells in the PD model. (F) FTH1 silencing downregulated MMP in 6-OHDA-stimulated cells. ${ }^{*} \mathrm{P}<0.05$, ${ }^{* *} \mathrm{P}<0.01$ and ${ }^{* * * * *} \mathrm{P}<0.0001$ vs. model group. Scale bar, $200 \mu \mathrm{m}$. Parkinson's disease; TH, tyrosine hydroxylase; GPX4, glutathione peroxidase 4; FTH1, ferritin heavy chain 1; 6-OHDA, 6-hydroxydopamine.

SN in the PD-affected brain (41). Therefore, the present study suggests that FTH1 plays a crucial role in reducing ferroptosis and alleviating dopaminergic neuron damage in PD.

Another important finding of the present study was that miR-335 promoted ferroptosis by inhibiting FTH1 expression in in vitro and in vivo PD models. Previously, it was unclear how miRNA regulates the level of ferroptosis in response to PD. In the present study, the results of RT-qPCR and DLR assay demonstrated that miR-335 specifically targets FTH1. The results of western blot analysis, cellular iron concentration assay, cellular ROS detection and MMP assays revealed that miR-335 aggravated ferroptosis and
PD injury by inhibiting FTH1 expression. These results support the finding that miRNAs act as crucial mediators of ferroptosis in PD by regulating protein translation (42-45). The findings of the present study provide new insight into the therapeutic potential of inhibiting miR-335 to increase the expression of FTH1, reduce $\mathrm{Fe}^{2+}$ concentration, and ultimately prevent ferroptosis in PD.

The results have important clinical implications. Firstly, miR-335 could be a potential biomarker in the diagnosis of PD and may represent a novel therapeutic target. However, clinical trials are required to further elucidate and validate this potential. Secondly, there are few therapies available 
for the treatment of PD using small molecules. The gold standard treatment currently used in patients with PD is orally-administered levodopa (L-dopa). However, L-dopa produces adverse drug reactions including dyskinesias and involuntary movement (46), resulting in increased financial and psychological burden for patients with PD. Molecular therapy currently presents a unique effect on neoplastic diseases, but is not generally used in PD. The results of the present study identified a potential therapy which may be used to protect against PD progression by inhibiting the expression of miR-335.

In conclusion, the present study demonstrates an important mechanism wherein miR-335 promotes ferroptosis in PD by inhibiting FTH1 expression in vitro and in vivo. These results provide a novel therapeutic approach for the treatment of PD, based on the regulation of the miR-335-FTH1-ferroptosis signaling pathway.

\section{Acknowledgements}

Not applicable.

\section{Funding}

The present study was supported by the Traditional Chinese Medicine Bureau of Guangdong Province, China (grant no. 20203010), and the Natural Science Foundation of Guangdong Province (grant no. 2020A151501325).

\section{Availability of data and materials}

The data and materials produced during the study can be obtained from the corresponding authors on reasonable request.

\section{Authors' contributions}

XLi performed most of the experiments, analyzed data and wrote most of the manuscript. WS designed the animal experiments and contributed to the manuscript. ZL contributed to the manuscript, assisted in the design of in vitro experiments, analyzed data and provided the reagents. YT contributed to the conception of the study and provided the reagents and materials. XLiu, SY, ZH, YJ, CZ and XH assisted with the in vivo experiments. MZ and DC conceived, designed and supervised the study. All authors read and approved the final manuscript.

\section{Ethics approval and consent to participate}

All animal experiments were approved by the Experimental Animal Care and Use Committee of Guangzhou University of Traditional Chinese medicine, and conducted in accordance with the guidelines for the care and use of experimental animals of the National Institutes of Health of the United States.

\section{Patient consent for publication}

Not applicable.

\section{Competing interests}

The authors declare no competing interests.

\section{References}

1. Lei P, Bai T and Sun Y: Mechanisms of ferroptosis and relations with regulated cell death: A review. Front Physiol 10: 139, 2019.

2. Muhoberac BB, Baraibar MA and Vidal R: Iron loading-induced aggregation and reduction of iron incorporation in heteropolymeric ferritin containing a mutant light chain that causes neurodegeneration. Biochim Biophys Acta 1812: 544-548, 2011.

3. Yang WS, SriRamaratnam R, Welsch ME, Shimada K, Skouta R, Viswanathan VS, Cheah JH, Clemons PA, Shamji AF, Clish CB, et al: Regulation of ferroptotic cancer cell death by GPX4. Cell 156: 317-331, 2014.

4. Yang WS and Stockwell BR: Ferroptosis: Death by lipid peroxidation. Trends Cell Biol 26: 165-176, 2016.

5. Maiorino M, Conrad M and Ursini F: GPx4, lipid peroxidation, and cell death: Discoveries, rediscoveries, and open issues. Antioxid Redox Signal 29: 61-74, 2018.

6. Seibt TM, Proneth B and Conrad M: Role of GPX4 in ferroptosis and its pharmacological implication. Free Radic Biol Med 133: 144-152, 2019.

7. Forcina GC and Dixon SJ: GPX4 at the crossroads of lipid homeostasis and ferroptosis. Proteomics 19: e1800311, 2019.

8. Xie Y, Hou W, Song X, Yu Y, Huang J, Sun X, Kang R and Tang D: Ferroptosis: Process and function. Cell Death Differ 23: 369-379, 2016.

9. Knovich MA, Storey JA, Coffman LG, Torti SV and Torti FM: Ferritin for the clinician. Blood Rev 23: 95-104, 2009.

10. Liu NQ, De Marchi T, Timmermans AM, Beekhof R, Trapman-Jansen AM, Foekens R, Look MP, van Deurzen CH, Span PN, Sweep FC, et al: Ferritin heavy chain in triple negative breast cancer: A favorable prognostic marker that relates to a cluster of differentiation 8 positive $\left(\mathrm{CD}^{+}\right)$effector T-cell response. Mol Cell Proteomics 13: 1814-1827, 2014.

11. Shpyleva SI, Tryndyak VP, Kovalchuk O, Starlard-Davenport A, Chekhun VF, Beland FA and Pogribny IP: Role of ferritin alterations in human breast cancer cells. Breast Cancer Res Treat 126: 63-71, 2011

12. Sammarco MC, Ditch S, Banerjee A and Grabczyk E: Ferritin L and $\mathrm{H}$ subunits are differentially regulated on a post-transcriptional level. J Biol Chem 283: 4578-4587, 2008.

13. Do Van B, Gouel F, Jonneaux A, Timmerman K, Gelé P, Pétrault M, Bastide M, Laloux C, Moreau C, Bordet R, et al: Ferroptosis, a newly characterized form of cell death in Parkinson's disease that is regulated by PKC. Neurobiol Dis 94: 169-178, 2016.

14. Tian Y, Lu J, Hao X, Li H, Zhang G, Liu X, Li X, Zhao C, Kuang W, Chen D and Zhu M: FTH1 inhibits ferroptosis through ferritinophagy in the 6-OHDA model of Parkinson's disease. Neurotherapeutics 17: 1796-1812, 2020.

15. Lu J, Liu X, Tian Y, Li H, Ren Z, Liang S, Zhang G, Zhao C, Li X, Wang T, et al: Moxibustion exerts a neuroprotective effect through antiferroptosis in Parkinson's disease. Evid Based Complement Alternat Med 2019: 2735492, 2019.

16. Moreau C, Duce JA, Rascol O, Devedjian JC, Berg D, Dexter D, Cabantchik ZI, Bush AI and Devos D; FAIRPARK-II study group: Iron as a therapeutic target for Parkinson's disease. Mov Disord 33: 568-574, 2018.

17. Bonn D: Pumping iron in Parkinson's disease. Lancet 347: 1614, 1996.

18. Guiney SJ, Adlard PA, Bush AI, Finkelstein DI and Ayton S: Ferroptosis and cell death mechanisms in Parkinson's disease. Neurochem Int 104: 34-48, 2017.

19. Biliński T, Krawiec Z, Liczmański A and Litwińska J: Is hydroxyl radical generated by the fenton reaction in vivo? Biochem Biophys Res Commun 130: 533-539, 1985.

20. Costello DJ, Walsh SL, Harrington HJ and Walsh CH: Concurrent hereditary haemochromatosis and idiopathic Parkinson's disease: A case report series. J Neurol Neurosurg Psychiatry 75: 631-633, 2004.

21. Miyajima H, Takahashi Y and Kono S: Aceruloplasminemia, an inherited disorder of iron metabolism. Biometals 16: 205-213, 2003. 
22. Borie C, Gasparini F, Verpillat P, Bonnet AM, Agid Y, Hetet G, Brice A, Dürr A and Grandchamp B; French Parkinson's disease genetic study group: Association study between iron-related genes polymorphisms and Parkinson's disease. J Neurol 249: 801-804, 2002

23. Rhodes SL, Buchanan DD, Ahmed I, Taylor KD, Loriot MA, Sinsheimer JS, Bronstein JM, Elbaz A, Mellick GD, Rotter JI and Ritz B: Pooled analysis of iron-related genes in Parkinson's disease: Association with transferrin. Neurobiol Dis 62: 172-178, 2014.

24. Ambros V: The functions of animal microRNAs. Nature 431: 350-355, 2004.

25. Bartel DP: MicroRNAs: Genomics, biogenesis, mechanism, and function. Cell 116: 281-297, 2004.

26. Bartel DP: MicroRNAs: Target recognition and regulatory functions. Cell 136: 215-233, 2009.

27. Bartel DP: Metazoan MicroRNAs. Cell 173: 20-51, 2018.

28. Su Y, Deng MF, Xiong W, Xie AJ, Guo J, Liang ZH, Hu B, Chen JG, Zhu X, Man HY, et al: MicroRNA-26a/death-associated protein kinase 1 signaling induces synucleinopathy and dopaminergic neuron degeneration in Parkinson's disease. Biol Psychiatry 85: 769-781, 2019.

29. Jin L, Wan W, Wang L, Wang C, Xiao J, Zhang F, Zhao J, Wang J, Zhan $\mathrm{C}$ and Zhong C: Elevated microRNA-520d-5p in the serum of patients with Parkinson's disease, possibly through regulation of cereloplasmin expression. Neurosci Lett 687: 88-93, 2018.

30. Asci R, Vallefuoco F, Andolfo I, Bruno M, De Falco L and Iolascon A: Trasferrin receptor 2 gene regulation by microRNA 221 in SH-SY5Y cells treated with MPP+ as Parkinson's disease cellular model. Neurosci Res 77: 121-127, 2013.

31. Rothblat DS, Schroeder JA and Schneider JS: Tyrosine hydroxylase and dopamine transporter expression in residual dopaminergic neurons: Potential contributors to spontaneous recovery from experimental Parkinsonism. J Neurosci Res 65: 254-266, 2001.

32. Deumens R, Blokland A and Prickaerts J: Modeling Parkinson's disease in rats: An evaluation of 6-OHDA lesions of the nigrostriatal pathway. Exp Neurol 175: 303-317, 2002.

33. Li Z, Lan X, Han R, Wang J, Huang Y, Sun J, Guo W and Chen $\mathrm{H}$ : miR-2478 inhibits TGF $\beta 1$ expression by targeting the transcriptional activation region downstream of the TGF $\beta 1$ promoter in dairy goats. Sci Rep 7: 42627, 2017.

34. Lee SJ, Jeong JH, Kang SH, Kang J, Kim EA, Lee J, Jung JH, Park HY and Chae YS: MicroRNA-137 inhibits cancer progression by targeting Del-1 in triple-negative breast cancer cells. Int J Mol Sci 20: 6162, 2019.

35. He H, Chen K, Wang F, Zhao L, Wan X, Wang L and Mo Z: miR-204-5p promotes the adipogenic differentiation of human adipose-derived mesenchymal stem cells by modulating DVL3 expression and suppressing Wnt/ $/$-catenin signaling. Int $\mathrm{J}$ Mol Med 35: 1587-1595, 2015.
36. Livak KJ and Schmittgen TD: Analysis of relative gene expression data using real-time quantitative PCR and the 2(-Delta Delta C(T)) method. Methods 25: 402-408, 2001.

37. Grayson M: Parkinson's disease. Nature 538: S1, 2016.

38. Dixon SJ, Lemberg KM, Lamprecht MR, Skouta R, Zaitsev EM Gleason CE, Patel DN, Bauer AJ, Cantley AM, Yang WS, et al: Ferroptosis: An iron-dependent form of nonapoptotic cell death. Cell 149: 1060-1072, 2012.

39. Jiang H, Wang J, Rogers J and Xie J: Brain iron metabolism dysfunction in Parkinson's disease. Mol Neurobiol 54: 3078-3101, 2017.

40. Powers KM, Smith-Weller T, Franklin GM, Longstreth WT Jr, Swanson PD and Checkoway H: Dietary fats, cholesterol and iron as risk factors for Parkinson's disease. Parkinsonism Relat Disord 15: 47-52, 2009.

41. Bellinger FP, Raman AV, Rueli RH, Bellinger MT, Dewing AS, Seale LA, Andres MA, Uyehara-Lock JH, White LR, Ross GW and Berry MJ: Changes in selenoprotein P in substantia nigra and putamen in Parkinson's disease. J Parkinsons Dis 2: 115-126, 2012.

42. Angelopoulou E, Paudel YN and Piperi C: $m i R-124$ and Parkinson's disease: A biomarker with therapeutic potential. Pharmacol Res 150: 104515, 2019.

43. De Gregorio R, Pulcrano S, De Sanctis C, Volpicelli F, Guatteo E, von Oerthel L, Latagliata EC, Esposito R, Piscitelli RM, Perrone-Capano C, et al: miR-34b/c regulates wnt 1 and enhances mesencephalic dopaminergic neuron differentiation. Stem Cell Rep 10: 1237-1250, 2018.

44. Luo M, Wu L, Zhang K, Wang H, Zhang T, Gutierrez L, O'Connell D, Zhang P, Li Y, Gao T, et al: miR-137 regulates ferroptosis by targeting glutamine transporter SLC1A5 in melanoma. Cell Death Differ 25: 1457-1472, 2018.

45. Sangokoya C, Doss JF and Chi JT: Iron-responsive miR-485-3p regulates cellular iron homeostasis by targeting ferroportin. PLoS Genet 9: e1003408, 2013.

46. Poewe W, Bergmann L, Kukreja P, Robieson WZ and Antonini A: Levodopa-carbidopa intestinal gel monotherapy: GLORIA registry demographics, efficacy, and safety. J Parkinsons Dis 9: 531-541, 2019.

This work is licensed under a Creative Commons Attribution-NonCommercial-NoDerivatives 4.0 International (CC BY-NC-ND 4.0) License. 